Properties of a Subclass of Meromorphic Univalent Functions By Using Multiplier transformation

Thamer Khalil Lieth A. Majed

\title{
Properties of a Subclass of Meromorphic Univalent Functions By Using Multiplier transformation
}

\author{
Thamer Khalil ${ }^{1}$ and Lieth A. Majed ${ }^{2}$ \\ ${ }^{1}$ College of Education - University of Al-Mustansirya \\ ${ }^{2}$ College of Science - University of Diyala
}

Received 1 April 2016

Accepted 18 May 2016

\begin{abstract}
$\underline{\text { Abstract }}$
In this present paper, we introduced and defined properties of a subclass of meromorphic univalent Functions defined by multiplier transformation in the puncture unit disk $\Delta^{*}=\{\mathrm{z} \in$ $\mathbb{C}: 0<|z|<1\}$. we obtain some properties, like, theorem of coefficient inequality, linear combination, extreme points and convex set.
\end{abstract}

Key words: coefficient inequality, linear combination, extreme points and convex set.

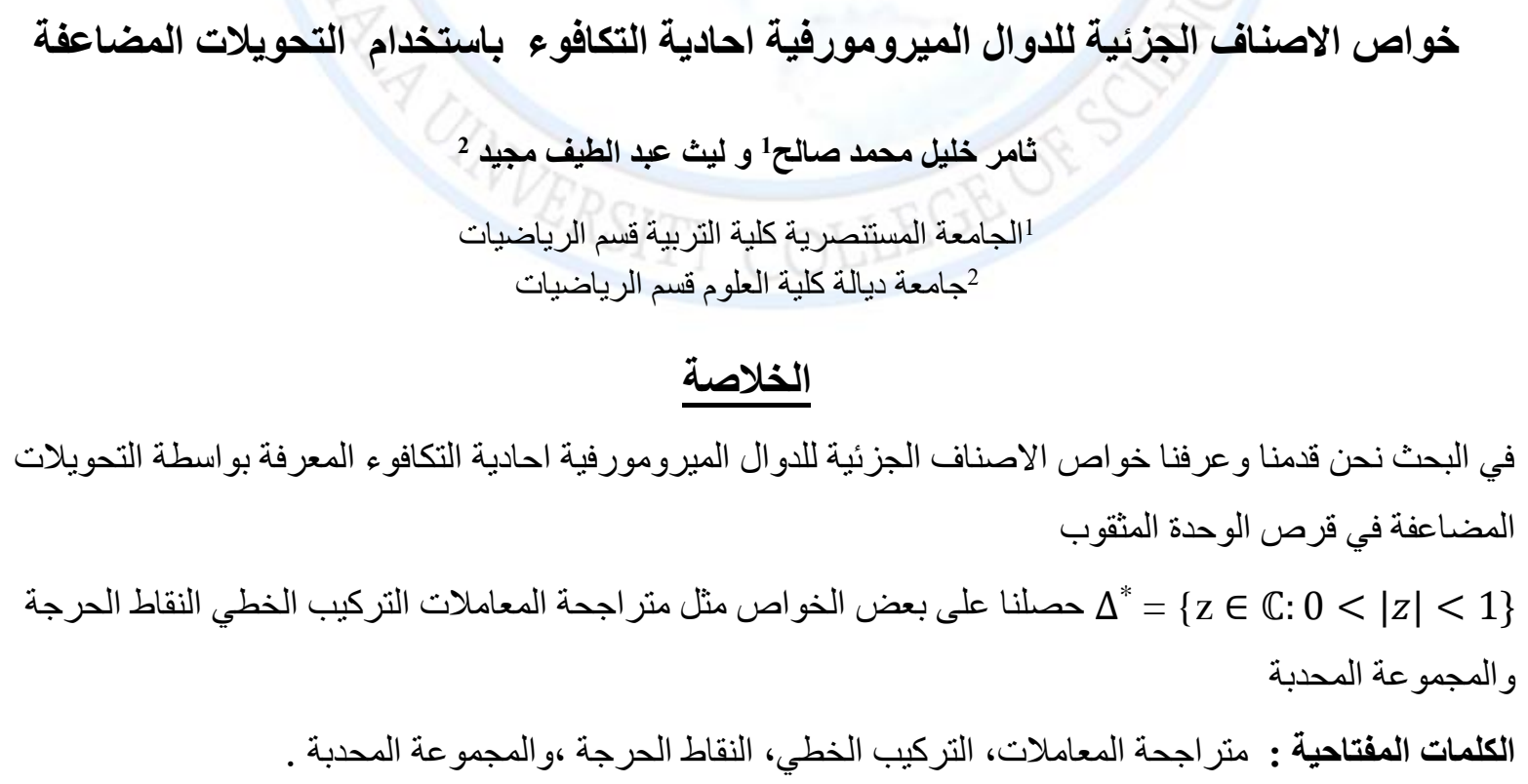




\section{Properties of a Subclass of Meromorphic Univalent Functions By Using Multiplier transformation}

Thamer Khalil Lieth A. Majed

\section{$\underline{\text { Introduction }}$}

Let $M F$ denote the class of meromorphic functions $f$ of the form

$$
h(z)=\frac{1}{z}+\sum_{m=1}^{\infty} a_{m} z^{m},
$$

defined on the punctured unit disk $\Delta^{*}=\{\mathrm{z} \in \mathbb{C}: 0<|\mathrm{z}|<1\}$.

Also, denote by $\Omega$ the subclass of $M F$ consisting of functions of the from

$$
h(z)=\frac{1}{z}+\sum_{m=1}^{\infty} a_{m} z^{m}, \quad\left(a_{m} \geq 0\right) .
$$

Now, we define on $\Omega$ multiplier transformation, we define the operator $L_{1}(r, \gamma)$ by the following infinite series when

$$
h(z)=\frac{1}{z}+\sum_{m=1}^{\infty} a_{m} z^{m}
$$

then

$$
L_{1}(r, \gamma) h(z)=\frac{1}{z}+\sum_{m=1}^{\infty}\left(\frac{m+\gamma}{1+\gamma}\right)^{r} a_{m} z^{m}(\gamma \geq 0) .
$$

The operator $L_{1}(r, \gamma)$ was considered by Cho and Srivastava [3] and Cho and Kim [2].

Definition (1): The function $k \in \Omega$ be of the form (2) is said to be in the new class $L_{1}(\tau, \alpha, \mu, r, \gamma)$ if it satisfies the following condition:

$$
\left|\frac{\frac{\frac{z^{2} \tau}{2}\left(L_{1}(r, \gamma)(h)(z)\right)^{\prime \prime}}{\left(L_{1}(r, \gamma)(h)(z)\right)}-\tau}{\alpha-\frac{\frac{z^{2} \alpha \mu}{2}\left(L_{1}(r, \gamma)(h)(z)\right)^{\prime \prime}}{\left(L_{1}(r, \gamma)(h)(z)\right)}}\right|<1,
$$

for $0<\mu \leq \frac{1}{2}, 0<\tau<1,0<\alpha<1$ and $r \in z=\{\ldots,-1,0,1, \ldots\}$. 


\section{Properties of a Subclass of Meromorphic Univalent Functions By Using Multiplier transformation}

Thamer Khalil

Lieth A. Majed

The following interesting geometric properties of this function subclass were studied by several authors for another classes, like, Darus [2], Atshan [1].

Now, we obtain the necessary and sufficient condition for a function $h$ to be in the class $L_{1}(\tau, \alpha, \mu, r, \gamma)$.

Theorem (1): Let $h \in \Omega$. Then $h \in L_{1}(\tau, \alpha, \mu, r, \gamma)$ if and only if

$$
\sum_{m=1}^{\infty}\left(\frac{m+\gamma}{1+\gamma}\right)^{r}\left(\frac{\tau}{2}\left(m^{2}-m\right)-\tau-\alpha+\frac{\alpha \gamma m}{2}(m-1)\right) a_{m} \leq \alpha(1-\mu)
$$

where $0<\mu \leq \frac{1}{2}, 0<\tau<1$, and $0<\alpha<1$.

The result is sharp for the fumctiom

$$
h(z)=\frac{1}{z}+\frac{\alpha(1-\mu)}{\left(\frac{m+\gamma}{1+\gamma}\right)^{r}\left(\frac{\tau}{2} m(m-1)-\tau-\alpha+\frac{\alpha \gamma m}{2}(m-1)\right)} z^{m}, m \in \mathbb{N} .
$$

Proof: For $|z|=1$, we have

$$
\begin{aligned}
& \quad\left|\frac{z^{2} \tau}{2}\left(L_{1}(r, \gamma)(h)(z)\right)^{\prime \prime}-\tau\left(L_{1}(r, \gamma)(h)(z)\right)\right| \\
& -\left|\alpha\left(L_{1}(r, \gamma)(h)(z)\right)-\frac{z^{2} \alpha \mu}{2}\left(L_{1}(r, \gamma)(h)(z)\right)^{\prime \prime}\right| \\
& =\left|\sum_{m=1}^{\infty}\left(\frac{\tau}{2}\left(m^{2}-m\right)-\tau\right)\left(\frac{m+\gamma}{1+\gamma}\right)^{r} a_{m} z^{m}\right| \\
& -\left|\alpha\left(z^{-1}+\sum_{m=1}^{\infty}\left(\frac{m+\gamma}{1+\gamma}\right)^{r} a_{m} z^{m}\right)-\frac{z^{2} \alpha \mu}{2}\left(2 z^{-3}+\sum_{m=1}^{\infty}\left(\frac{m+\gamma}{1+\gamma}\right)^{r} a_{n} m(m-1) z^{m-2}\right)\right| \\
& =\left|\sum_{m=1}^{\infty}\left(\frac{\tau}{2} m(n-1)-\tau\right)\left(\frac{m+\gamma}{1+\gamma}\right)^{r} a_{m} z^{m}\right|
\end{aligned}
$$




\section{Properties of a Subclass of Meromorphic Univalent Functions By Using Multiplier transformation}

Thamer Khalil

Lieth A. Majed

$$
\begin{aligned}
& \quad-\left|\alpha z^{-1}+\alpha \sum_{m=1}^{\infty}\left(\frac{m+\gamma}{1+\gamma}\right)^{r} a_{m} z^{m}-\alpha \mu z^{-1}-\sum_{m=1}^{\infty} \frac{\alpha}{2} \mu m\left(\frac{m+\gamma}{1+\gamma}\right)^{r} a_{m} z^{m}\right| \\
& =\left|\sum_{m=1}^{\infty}\left(\frac{\tau}{2} m(m-1)-\tau\right)\left(\frac{m+\gamma}{1+\gamma}\right)^{r} a_{m} z^{m}\right| \\
& \quad-\left|(\alpha-\alpha \mu) z^{-1}-\sum_{m=1}^{\infty}\left(-\alpha+\frac{\alpha \mu m}{2}(m-1)\right)\left(\frac{m+\gamma}{1+\gamma}\right)^{r} a_{m} z^{m}\right| \\
& \leq \sum_{m=1}^{\infty}\left(\frac{\tau}{2} m(m-1)-\tau\right)\left(\frac{m+\gamma}{1+\gamma}\right)^{r} a_{m}-(\alpha-\alpha \gamma)+\sum_{m=1}^{\infty}\left(-\alpha+\frac{\alpha \mu m}{2}(m-1)\right)\left(\frac{m+\gamma}{1+\gamma}\right)^{r} a_{m} \\
& =\sum_{m=1}^{\infty}\left(\frac{m+\gamma}{1+\gamma}\right)^{r}\left(\frac{\tau}{2} m(m-1)-\tau-\alpha+\frac{\alpha \gamma m}{2}(m-1)\right) a_{m}-\alpha(1-\mu) \leq 0 .
\end{aligned}
$$

by hypothesis. Hence, $h \in L_{1}(\tau, \alpha, \mu, r, \gamma)$.

Conversely, assume that $h \in L_{1}(\tau, \alpha, \mu, r, \gamma)$, then from (4), we have

$$
\begin{aligned}
& \left|\frac{\frac{\frac{z^{2} \tau}{2}\left(L_{1}(r, \gamma)(h)(z)\right)^{\prime \prime}}{\left(L_{1}(r, \gamma)(h)(z)\right)}-\tau}{\alpha-\frac{\frac{z^{2} \alpha \mu}{2}\left(L_{1}(r, \gamma)(h)(z)\right)^{\prime \prime}}{\left(L_{1}(r, \gamma)(h)(z)\right)}}\right| \\
& =\left|\frac{\sum_{m=1}^{\infty}\left(\frac{\tau}{2}\left(m^{2}-m\right)-\tau\right)\left(\frac{m+\gamma}{1+\gamma}\right)^{r} a_{m} z^{m}}{(\alpha-\alpha \mu) z^{-1}-\sum_{m=1}^{\infty}\left(-\alpha+\frac{\alpha \mu m}{2}(m-1)\right)\left(\frac{m+\gamma}{1+\gamma}\right)^{r} a_{m} z^{m}}\right|<1
\end{aligned}
$$

Since $\operatorname{Re}(\mathrm{z}) \leq|z| \forall \mathrm{z}\left(\mathrm{z} \in \Delta^{*}\right)$, we get

$\operatorname{Re}\left\{\frac{\sum_{m=1}^{\infty}\left(\frac{\tau}{2}\left(m^{2}-m\right)-\tau\right)\left(\frac{m+\gamma}{1+\gamma}\right)^{r} a_{m} z^{m}}{(\alpha-\alpha \mu) z^{-1}-\sum_{m=1}^{\infty}\left(-\alpha+\frac{\alpha \mu m}{2}(m-1)\right)\left(\frac{m+\gamma}{1+\gamma}\right)^{r} a_{m} z^{m}}\right\} \leq 1$

We can choose value of $z$ on the real axis $\frac{z^{2}\left(L_{1}(r, \gamma)(h)(z)\right)^{\prime \prime}}{\left(L_{1}(r, \gamma)(h)(z)\right)} \in \operatorname{Re}$. 


\section{Properties of a Subclass of Meromorphic Univalent Functions By Using Multiplier transformation}

$$
\begin{aligned}
& \sum_{m=1}^{\infty}\left(\frac{\tau}{2}\left(m^{2}-m\right)-\tau\right)\left(\frac{m+\gamma}{1+\gamma}\right)^{r} a_{m} z^{m} \\
& \quad \leq(\alpha-\alpha \mu) z^{-1}-\sum_{m=1}^{\infty}\left(-\alpha+\frac{\alpha \mu m}{2}(-1+m)\right)\left(\frac{m+\gamma}{1+\gamma}\right)^{r} a_{m} z^{m}
\end{aligned}
$$

Let $\operatorname{Re} \mathrm{z} \rightarrow 1^{-}$

$$
\begin{aligned}
\sum_{m=1}^{\infty}\left(\frac{\tau}{2}\left(m^{2}-m\right)\right. & -\tau)\left(\frac{m+\gamma}{1+\gamma}\right)^{r} a_{m} \\
& \leq-\sum_{m=1}^{\infty}\left(\frac{n+\gamma}{1+\gamma}\right)^{r}\left(\frac{\tau}{2}\left(m^{2}-m\right)-\tau-\alpha+\frac{\alpha \gamma m}{2}(-1+m)\right) a_{m}+\alpha(1-\mu) .
\end{aligned}
$$

we can write (6) as

$$
\sum_{m=1}^{\infty}\left(\frac{m+\gamma}{1+\gamma}\right)^{r}\left(\frac{\tau}{2}\left(m^{2}-m\right)-\tau-\alpha+\frac{\alpha \gamma m}{2}(-1+m)\right) a_{m} \leq \alpha(1-\mu) .
$$

Finally,

$$
h_{m}(z)=z^{-1}+\frac{\alpha(1-\mu)}{\left(\frac{m+\gamma}{1+\gamma}\right)^{r}\left(\frac{\tau}{2}\left(m^{2}-m\right)-\tau-\alpha+\frac{\alpha \gamma m}{2}(n-1)\right)} z^{n}, m=1,2, \ldots
$$

Corollary (1): Let $h \in L_{1}(\tau, \alpha, \mu, r, \gamma)$ Then

$$
a_{\mathrm{m}} \leq \frac{\alpha(1-\mu)}{\left(\frac{m+\gamma}{1+\gamma}\right)^{r}\left(\frac{\tau}{2}\left(m^{2}-m\right)-\tau-\alpha+\frac{\alpha \gamma m}{2}(m-1)\right)}, m=1,2, \ldots .
$$

In the following theorem, we will show the class $L_{1}(\tau, \alpha, \mu, r, \gamma)$ is linear combination

Theorem (2): Let

$$
h_{i}(z)=z^{-1}+\sum_{m=1}^{\infty} a_{m, i} z^{m} \in L_{1}(\tau, \alpha, \mu, r, \gamma) i \in\{1,2, \ldots, \ell\} \text { and }
$$

$0<c_{i}<1$ 


\section{Properties of a Subclass of Meromorphic Univalent Functions By Using Multiplier transformation}

Thamer Khalil Lieth A. Majed

Such that

$$
\sum_{i=1}^{\ell} c_{i}=1
$$

Then

$$
H=\sum_{i=1}^{\ell} c_{i} h_{i}(z)
$$

is also in the class $L_{1}(\tau, \alpha, \mu, r, \gamma)$.

Proof: By Theorem (1) for every $i \in\{1,2, \ldots, \ell\}$ we have

$$
\sum_{m=1}^{\infty} \frac{\left(\frac{m+\gamma}{1+\gamma}\right)^{r}\left(\frac{\tau}{2}\left(m^{2}-m\right)-\tau-\alpha+\frac{\alpha \gamma m}{2}(-1+m)\right)}{\alpha(1-\mu)} a_{m, i} \leq 1
$$

Since

$$
\begin{gathered}
H(z)=\sum_{i=1}^{\ell} c_{i} h_{i}(z)=\sum_{i=1}^{\ell} c_{i}\left(\mathrm{z}^{-1}+\sum_{\mathrm{m}=1}^{\infty} a_{\mathrm{m}, \mathrm{i}} \mathrm{z}^{\mathrm{m}}\right) \\
=\frac{1}{z}+\sum_{\mathrm{m}=1}^{\infty}\left(\sum_{i=1}^{\ell} c_{i} a_{m, i}\right) \mathrm{z}^{\mathrm{m}}
\end{gathered}
$$

Therefore

$$
\sum_{m=1}^{\infty} \frac{\left(\frac{m+\gamma}{1+\gamma}\right)^{r}\left(\frac{\tau}{2}\left(m^{2}-m\right)-\tau-\alpha+\frac{\alpha \gamma m}{2}(m-1)\right)}{\alpha(1-\mu)}\left(\sum_{i=1}^{\ell} c_{i} a_{m, i}\right)
$$




\section{Properties of a Subclass of Meromorphic Univalent Functions By Using Multiplier transformation}

Thamer Khalil

Lieth A. Majed

$$
\begin{gathered}
=\sum_{i=1}^{\ell} c_{i}\left(\sum_{m=1}^{\infty} \frac{\left(\frac{n+\gamma}{1+\gamma}\right)^{r}\left(\frac{\tau}{2}\left(m^{2}-m\right)-\tau-\alpha+\frac{\alpha \gamma n}{2}(-1+m)\right)}{\alpha(1-\mu)} a_{m, i}\right) \\
\leq \sum_{i=1}^{\ell} c_{i}=1
\end{gathered}
$$

Hence $H \in L_{1}(\tau, \alpha, \mu, r, \gamma)$ and the proof is complete.

In the following theorem, we obtain the extreme points of the class $L_{1}(\tau, \alpha, \mu, r, \gamma)$.

Theorem (3): Let $h_{0}(z)=\frac{1}{z}$ and

$$
h_{m}(z)=z^{-1}+\frac{\alpha(1-\mu)}{\left(\frac{m+\gamma}{1+\gamma}\right)^{r}\left(\frac{\tau}{2}\left(m^{2}-m\right)-\tau-\alpha+\frac{\alpha \gamma m}{2}(-1+m)\right)} z^{m},(m \geq 1) .
$$

Then $h \in L_{1}(\tau, \alpha, \mu, r, \gamma)$, if and only if

$$
h(z)=w_{0} h_{0}(z)+\sum_{m=1}^{\infty} w_{n} h_{n}(z), \quad\left(w_{m} \geq 0, w_{0}+\sum_{m=1}^{\infty} w_{m}=1\right) .
$$

Proof: Suppose that

$$
\begin{gathered}
h(z)=w_{0} h_{0}(z)+\sum_{m=1}^{\infty} w_{m} h_{m}(z) \\
h(z)=\frac{1}{z}+\sum_{m=1}^{\infty} \frac{\alpha(1-\mu)}{\left(\frac{m+\gamma}{1+\gamma}\right)^{r}\left(\frac{\tau}{2}\left(m^{2}-m\right)-\tau-\alpha+\frac{\alpha \gamma m}{2}(-1+m)\right)} z^{m}
\end{gathered}
$$

then 


\section{Properties of a Subclass of Meromorphic Univalent Functions By Using Multiplier transformation}

\section{Thamer Khalil Lieth A. Majed}

$$
\begin{gathered}
\sum_{m=1}^{\infty} \frac{\left(\frac{m+\gamma}{1+\gamma}\right)^{r}\left(\frac{\tau}{2}\left(m^{2}-m\right)-\tau-\alpha+\frac{\alpha \gamma m}{2}(m-1)\right)}{\alpha(1-\mu)} \\
\times w_{n} \frac{\alpha(1-\mu)}{\left(\frac{m+\gamma}{1+\gamma}\right)^{r}\left(\frac{\tau}{2}\left(m^{2}-m\right)-\tau-\alpha+\frac{\alpha \gamma m}{2}(m-1)\right)} \\
\quad=\sum_{m=1}^{\infty} w_{m}=1-w_{0} \leq 1 .
\end{gathered}
$$

So by Theorem (1), $h \in L_{1}(\tau, \alpha, \mu, r, \gamma)$. Conversly, we suppose $h \in L_{1}(\tau, \alpha, \mu, r, \gamma)$. By (8), we have

$$
a_{\mathrm{m}} \leq \frac{\alpha(1-\mu)}{\left(\frac{m+\gamma}{1+\gamma}\right)^{r}\left(\frac{\tau}{2}\left(m^{2}-m\right)-\tau-\alpha+\frac{\alpha \gamma m}{2}(m-1)\right)}, m \geq 1
$$

Setting

$$
w_{m}=\frac{\left(\frac{m+\gamma}{1+\gamma}\right)^{r}\left(\frac{\tau}{2}\left(m^{2}-m\right)-\tau-\alpha+\frac{\alpha \gamma m}{2}(n-1)\right)}{\alpha(1-\mu)} a_{m}, \quad m \geq 1,
$$

and

$$
w_{0}=1-\sum_{m=1}^{\infty} w_{m}
$$

Then

$$
h(z)=w_{0} h_{0}(z)+\sum_{m=1}^{\infty} w_{m} h_{m}(z)
$$

Then 


\section{Properties of a Subclass of Meromorphic Univalent Functions By Using Multiplier transformation}

\section{Thamer Khalil I Lieth A. Majed}

$$
\begin{gathered}
h(z)=\frac{1}{z}+\sum_{m=1}^{\infty} a_{m} z^{m} \\
h(z)=\frac{1}{z}+\sum_{m=1}^{\infty} \frac{\alpha(1-\mu) w_{n}}{\left(\frac{m+\gamma}{1+\gamma}\right)^{r}\left(\frac{\tau}{2}\left(m^{2}-m\right)-\tau-\alpha+\frac{\alpha \gamma m}{2}(-1+m)\right)} z^{m} \\
=\frac{1}{z}+\sum_{m=1}^{\infty}\left(h_{m}-z^{-1}\right) w_{m} \\
=\frac{1}{z}\left(1-\sum_{m=1}^{\infty} w_{m}\right)+\sum_{m=1}^{\infty} w_{m} h_{m} \\
=z^{-1} w_{0}+\sum_{m=1}^{\infty} w_{m} h_{m} \\
h(z)=w_{0} h_{0}(z)+\sum_{m=1}^{\infty} w_{m} h_{m}(z) .
\end{gathered}
$$

In the following theorem, we will prove the class $L_{1}(\tau, \alpha, \mu, r, \gamma)$, is a convex set.

Theorem (4): The class $L_{1}(\tau, \alpha, \mu, r, \gamma)$ is convex set.

Proof: Let $f_{1}$ and $f_{2}$ be the arbitrary elements of the class $L_{1}(\tau, \alpha, \mu, r, \gamma)$. Then for every $\mathrm{k}$ $(0<k<1)$, we will show that

$(1-Q) h_{1}+Q h_{2} \in L_{1}(\tau, \alpha, \mu, r, \gamma)$

Thus, we have

$$
(1-Q) h_{1}+Q h_{2}=\frac{1}{z}-\sum_{m=1}^{\infty}\left[(1-Q) a_{m}+Q b_{m}\right] z^{m}
$$

Hence, 


\section{Properties of a Subclass of Meromorphic Univalent Functions By Using Multiplier transformation}

Thamer Khalil
Lieth A. Majed

$$
\begin{gathered}
\sum_{m=1}^{\infty}\left(\frac{m+\gamma}{1+\gamma}\right)^{r}\left(\frac{\tau}{2}\left(m^{2}-m\right)-\tau-\alpha+\frac{\alpha \gamma m}{2}(m-1)\right)\left[(1-Q) a_{m}+Q b_{m}\right] \\
=(1-Q) \sum_{m=1}^{\infty}\left(\frac{m+\gamma}{1+\gamma}\right)^{r}\left(\frac{\tau}{2}\left(m^{2}-m\right)-\tau-\alpha+\frac{\alpha \gamma m}{2}(m-1)\right) a_{m} \\
+Q \sum_{m=1}^{\infty}\left(\frac{m+\gamma}{1+\gamma}\right)^{r}\left(\frac{\tau}{2}\left(m^{2}-m\right)-\tau-\alpha+\frac{\alpha \gamma m}{2}(m-1)\right) b_{m} \\
\leq(1-Q) \alpha(1-\mu)+\alpha(1-\mu) Q=\alpha(1-\mu) .
\end{gathered}
$$

\section{$\underline{\text { References }}$}

1. W. G. Atshan, Subclass of meromorphic functions with positive coefficients defined by Ruscheweyh derivative II,J. Surrveys in Mathematics and its Aplictions, 3(2008), 6777.

2. N. E. Cho and T. H. Kim, Multiplier transformations and strongly close-to-convex functions, Bulletin of the Korean Mathematical Society, 40 (3) (2003), 399-410.

3. N. E. Cho and H.M. Srivastava, Argument estimates of certain analytic functions defined by a class of multipier transformations, Math. Comput. Modelling, 37(12)(2003),39-49.

4. S. Najafzadeh and A. Ebadian, Convex family of meromorphically multivalent functions on connected sets, Math. Com. Mod., 57(2013), 301-305. 